\title{
Grußwort Buchprojekt Hernienschule
}

Das dreiarmige Weiterbildungskonzept „Hernie kompakt“, „Hernie konkret“ und „Hernie komplex“ im Rahmen der Hernienschule der Deutschen Herniengesellschaft (DHG) ist bislang weltweit einmalig. Die Ziele des 3-tägigen Basiskurses „Hernie Kompakt“ sind am ersten Tag:

1. Vertiefung der Kenntnisse der Anatomie von Leistenregion und Bauchdecke durch Vorträge, in der Ultraschalldiagnostik und beim Operieren am Cadaversitus.

2. Im Modul „Modelltraining“ überprüfen der handwerklichen Fähigkeiten v. a. beim intrakorporalen Nähen.

3. Demonstration der Standardtechniken: offen und laparo-endoskopisch, einzelne Operationsschritte werden vom Kursteilnehmer selbständig durchgeführt.

Der zweite Tag wird im Operationssaal verbracht und am dritten Tag wird in Vorträgen detailliert auf die Techniken, ihre Ergebnisse sowie deren wissenschaftliche Begründungen eingegangen. Im Weiterbildungsarm „Hernie Konkret“ werden die speziellen Operationstechniken beim Besuch in einem Hernienzentrum vom entsprechenden Experten demonstriert und im Kurssegment „Hernie Komplex“ auf die schwierige/ komplexe Hernie sowie auf das Komplikationsmanagement eingegangen.

Ohne Zweifel ist der Nutzen eines derart aufwendigen Weiterbildungssystems wesentlich größer, wenn der Teilnehmer sich bereits auf den Kurs vorbereiten kann und über gewisse Grundkenntnisse verfügt. Es kann nicht angehen, dass, wie das bei vielen der teilnehmenden jungen Chirurgen der Fall ist, die Neuroanatomie der Leistenregion noch völlig unbekannt ist. Der Nutzen des Kurses ist in einem derartigen Fall deutlich begrenzt. Das Gleiche gilt auch für seine Nachbearbeitung bzw. für die Nachhaltigkeit. Es ist nur allzu menschlich, wenn nach Rückkehr des Teilnehmers in die tägliche Routine seiner Klinik das Gesehene und Erlernte rasch in Vergessenheit gerät oder geraten muss, da der Chef oder die Oberärzte andere Auffassungen und eigene Standards haben und nicht bereit sind, Neues hinzuzulernen. Hier nun ist der Einsatzpunkt des vorliegenden Kompendiums, es soll der Vor- und Nachbereitung des Kurssystems dienen, sodass der Teilnehmer einerseits einen optimalen Nutzen hat, andererseits aber auch für die hoffentlich anschließenden Diskussionen in der eigenen Klinik einen Leitfaden an der Hand hat. Das sehr lesenswerte Kompendium sollte daher nicht nur für den jungen Chirurgen, sondern auch für seine leitenden Ärzte eine Pflichtlektüre sein, letztlich mit dem Ziel, die Versorgung der Patienten entsprechend dem aktuellen Standard zu optimieren. 
Es ist mir an dieser Stelle ein Anliegen, den drei Initiatoren dieses innovativen Weiterbildungskonzeptes, den Doktoren Wolfgang Reinpold, Bernd Stechemesser und Ralph Lorenz für ihr außerordentliches Engagement ganz herzlich zu danken.

Möge das Kompendium zur Standardlektüre für alle an der Hernienchirurgie interessierten Kollegen werden.

Professor Dr. med. Dr. h.c. mult. Reinhard Bittner, FRCS, FICS 